\title{
BMJ Open Quality Wessex Acute Frailty Audit: applying quality improvement methodology to design and implement a regional frailty audit using a collaborative, multiprofessional approach
}

\author{
Lucy Anne Lewis (D) , ${ }^{1}$ Teresa Corbett, ${ }^{2}$ Kerry Burrows, ${ }^{3}$ Claire Spice, ${ }^{3}$ \\ Cheryl Davies, ${ }^{4}$ Kathy Wallis ${ }^{4}$
}

To cite: Lewis LA, Corbett T, Burrows K, et al. Wessex Acute Frailty Audit: applying quality improvement methodology to design and implement a regional frailty audit using a collaborative, multiprofessiona approach. BMJ Open Quality 2020;9:e000870. doi:10.1136/ bmjoq-2019-000870

Received 31 October 2019 Revised 2 January 2020 Accepted 14 January 2020

A) Check for updates

(c) Author(s) (or their employer(s)) 2020. Re-use permitted under CC BY-NC. No commercial re-use. See rights and permissions. Published by BMJ.

${ }^{1}$ Consultant Practitioner Development Programme, Health Education England Wessex, Winchester, UK

${ }^{2}$ Department of Health Sciences, University of Southampton,

Southampton, UK

${ }^{3}$ Medcine for Older People, Portsmouth Hospitals NHS Trust, Portsmouth, UK

${ }^{4}$ Healthy Ageing Programme, Wessex Academic Health Science Network, Southampton, UK

Correspondence to

Mrs Lucy Anne Lewis;

l.lewis2@nhs.net

\section{ABSTRACT}

Introduction An acute hospital stay increases the risk of negative outcomes for those living with frailty. This paper describes the application of quality improvement methodology to design and implement a regional audit to gain an understanding of care provision.

Methods Small scale tests of change (Plan-Do-StudyAct cycles) were used to design the audit structure and questions. Data collectors met face to face with 2-3 multiprofessional clinicians on 58 wards in 10 hospitals across the region, using an electronic tool to gather data. Outcomes were analysed manually in Excel by extracting from the electronic audit tool.

Results 58 wards across 10 hospitals participated in the audit, which identified three key themes: lack of awareness and frailty training outside medicine for older people specialties, and significant variability of both frailty identification and comprehensive geriatric assessment. Conclusion Combining quality improvement methodology with a collaborative, regional approach to design and implementation of a frailty audit creates a reliable tool ensuring all stakeholders are considering improvement from the outset. The results have facilitated an agreed regional approach on how best to use local resources to improve and standardise frailty care provision. By highlighting areas of good practice and significant gaps in frailty identification, personalised care planning and hospital wide provision of frailty training, this region of the UK will now be able to drive up standards of care.

\section{INTRODUCTION}

An acute hospital admission compromises the physical, psychological and social wellbeing of people living with frailty and can increase mortality. ${ }^{12}$ Clinicians not equipped with the knowledge to identify a person living with frailty, assess their individual needs and provide appropriate support have the potential to increase the risk of negative outcomes for these people. Hospital-wide identification and management of frailty is inconsistent. ${ }^{34}$ Root causes may include educational needs and service integration leading to the inability to adjust and share supportive care plans appropriately during admission and following discharge. There was anecdotal inconsistency between organisations in our region affecting equity and quality of care delivery, and we had no meaningful method of sharing learning and good practice. Expert group members had substantial experience with Sentinel Stroke National Audit Programme (SSNAP), which has been voted the most effective national clinical audit in the UK for nine consecutive years by healthcare professionals. ${ }^{5}$ In addition to its function as an audit tool, the questions included within SSNAP have been used as a mechanism by which to set the standards of stroke care that services should aspire to. The long-term aim of this project is to create a similar audit structure that could be used to continually improve the standards of care provided for people living with frailty attending our acute hospitals.

\section{AVAILABLE KNOWLEDGE}

A National Institute of Health Research (NIHR) comprehensive themed review of 53 studies, which focused on implementing methods to improve how hospital healthcare professionals care for older people living with frailty who are admitted to an acute hospital, was published in 2017. ${ }^{6}$ This provided evidence that if a person is identified as living with frailty, a comprehensive geriatric assessment (CGA) can reduce harms, mortality and admission to residential care. CGA identifies key issues affecting an individual living with frailty. It is a multidimensional and interprofessional process that leads to an integrated person-centred plan for treatment, therapy and review. ${ }^{78}$ The identification of frailty and subsequent CGAs and personalised planning can improve outcomes for those living with 
frailty in the acute hospital. CGA has been associated with reducing hospital length of stay, in patient-related complications and can lead to cost savings. ${ }^{89}$

The NIHR comprehensive themed review also revealed there was inadequate transfer of information between social care, community and acute care providers. When services and support are not integrated, delayed transfers of care, readmissions and suboptimal care can result. ${ }^{10}$ There is a need to deliver integrated support for older people rather than focusing on episodic treatment for acute illness alone. ${ }^{11}$ Only $42 \%$ of acute hospitals undertake early CGA despite substantial evidence to support that assessing and coordinating care using CGA in hospitals improves outcomes for people living with frailty. ${ }^{11} 12$ The ability of the multiprofessional team to identify frailty syndromes is low in staff outside specialist older people's services even though they are markers of poor outcomes. ${ }^{13}$

\section{RATIONALE}

Therefore, because of these findings and recommendations, planning of the Wessex Acute Frailty Audit commenced. It is important to describe the methods involved because interventions are often not clearly described in sufficient detail to allow for replication. ${ }^{14}$ This paper outlines the process to allow readers to understand how the application of quality improvement (QI) methodology can be used to design and implement a regional audit. There is a need to define set standards for frailty best practice in our region. Continual use of the audit cycle to measure effectiveness locally or nationally has been evidenced to result in improvements in older people's healthcare that are progressive and sustainable. ${ }^{15}$ By gaining a regional understanding of care provision, our aim was to identify areas of good practice and significant gaps in frailty identification, personalised care planning and hospital-wide provision of frailty training. This would enable understanding of key development needs and a regional approach to drive up standards of care. As the subject matter has the potential to be subjective, the model of clinical audit was selected to establish objectively how each organisation was performing. Areas for improvement would subsequently be identified and presented to executive staff at participating organisations during a whole day workshop. The aim of this workshop was to allow staff the opportunity to develop their own unique QI plans to improve frailty care based on their individual hospital audit results.

\section{Aim}

The aim of this study was to outline the rationale, decisionmaking processes, methods and findings that led to the design and implementation of a regional audit.

\section{Methods}

Context

The purpose of the Wessex Acute Frailty Audit was to benchmark services against evidenced best practice for the identification of people living with frailty in hospital, with the aim to reduce the unintended variation of care, thus increasing the quality of care across the region.

Objectives of the audit using The Model for Improvement. ${ }^{15}$

What are we trying to accomplish?

To gain a regional understanding of care provision to identify areas of good practice and significant gaps in frailty identification, personalised care planning and hospital-wide provision of frailty training.

How will we know that a change is an improvement?

Adequate audit participation from each trust will confirm if audit design and implementation using QI methodology is an effective method to gain knowledge.

What change can we make that will result in an improvement?

Use the data generated by audit results to improve and standardise care of older people living with frailty who are admitted to acute hospitals.

\section{PERSONNEL INVOLVED}

The Wessex Academic Health Science Network (AHSN) Healthy Ageing programme brought together a multiprofessional group of frailty experts. Each senior clinician contributed a wealth of experience, skill and knowledge in the specialty of older people's healthcare. They represented acute hospitals from throughout the Wessex region. Occupational therapists, physiotherapists, a pharmacist, nurses, geriatricians and a National Institute Health Research (NIHR) Dissemination Centre clinical advisor. Members ensured good communication with all nine organisations (10 hospitals) to achieve adequate engagement and participation. The core group were the key decision makers on audit issues led by the Wessex AHSN associate director for strategic programmes and included a trust frailty pathway lead, a frailty pharmacist and three geriatricians. In June 2018, a consultant nurse trainee joined as clinical coordinator, in addition to two senior specialist therapists (occupational therapist and physiotherapist), all with extensive acute frailty experience. The Healthy Ageing programme manager was appointed in October 2018. Figure 1 Demonstrates the timeline of audit design, planning and implementation phases.

\section{USE OF QI METHODOLOGY}

QI was used in the design and implementation phase to inform the focus of future projects as the umbrella in which the audit would sit under rather than viewing it separately from the anticipated service improvement projects that would result from audit findings. ${ }^{16} 17$ Local context could be lacking when auditing high-level outcome measures advising the inclusion of key stakeholders to promote the identification of problems specific to the local context. ${ }^{18}$ QI can assist in focusing on processes within each locality. A process map was created that displays the areas of each hospital where data were to be collected in relation to admission and discharge pathways. This included 


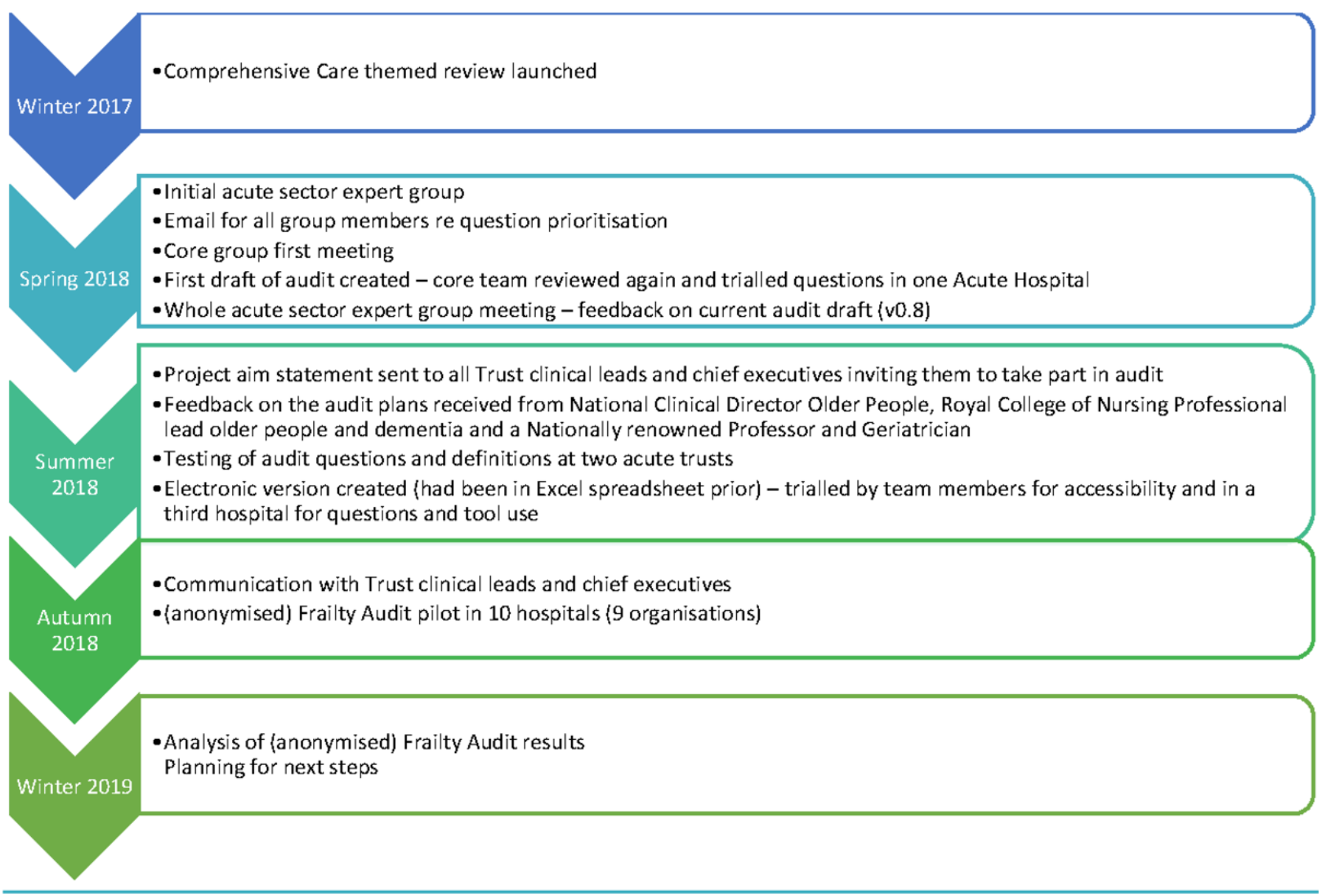

Figure 1 Timeline of audit design and implementation activity.

emergency department, acute medical units, acute frailty units, medicine for older people wards, general surgery, general medicine and trauma and orthopaedic units. As this was a pilot audit, the decision was taken to focus on this selection of wards in each hospital rather than auditing all specialities at this stage.

\section{Identification of barriers and facilitators of the audit}

There were many factors identified from the outset that could threaten the success of audit. To mitigate this, a project initiation document was written, including potential risks and benefits. A fishbone diagram grouped these issues together under the headings of stakeholders, design, delivery and geography.

\section{Strategies to overcome identified barriers to the audit using small scale tests of change}

The audit design was complemented by the addition of the Model for Improvement Plan-Do-Study-Act (PDSA) test cycles. Each series of PDSA cycles tested the hypothesis of each intervention to improve the process through applying this method of learning with small, rapid incremental steps ${ }^{19}$ Each PDSA cycle moved the audit design forward, allowed collaborative communication and supported engagement. Taking a PDSA approach also ensured the core team paused, considered and reviewed before planning the next phase enabling adaptation of the questions and review of our delivery. The PDSA cycles are illustrated in figure 2 .

\section{Audit question development}

Audit question development spanned over 7 months. Key findings from the NIHR themed review were discussed at the initial acute sector frailty expert group workshop. Four key topics were chosen, and the group began to devise questions that would provide information about these:

- Identifying people living with frailty.

- Supporting people living with frailty in hospital.

- Sharing of personalised care plans and transitioning to the next stage of care.

- Education, training and frailty awareness of clinical staff.

Using ideas collated from the initial acute expert group workshop, all members were asked to prioritise questions in each of these sections for inclusion in the audit. A draft audit was designed, and a geriatrician trialled in one hospital for feasibility before the next group meeting to discuss feedback. Following this expert group meeting, the small core group was formed, whose focus was to refine the audit questions and design. Feedback was also gathered from three older persons' healthcare national leads to ensure a broader perspective on the audit. 


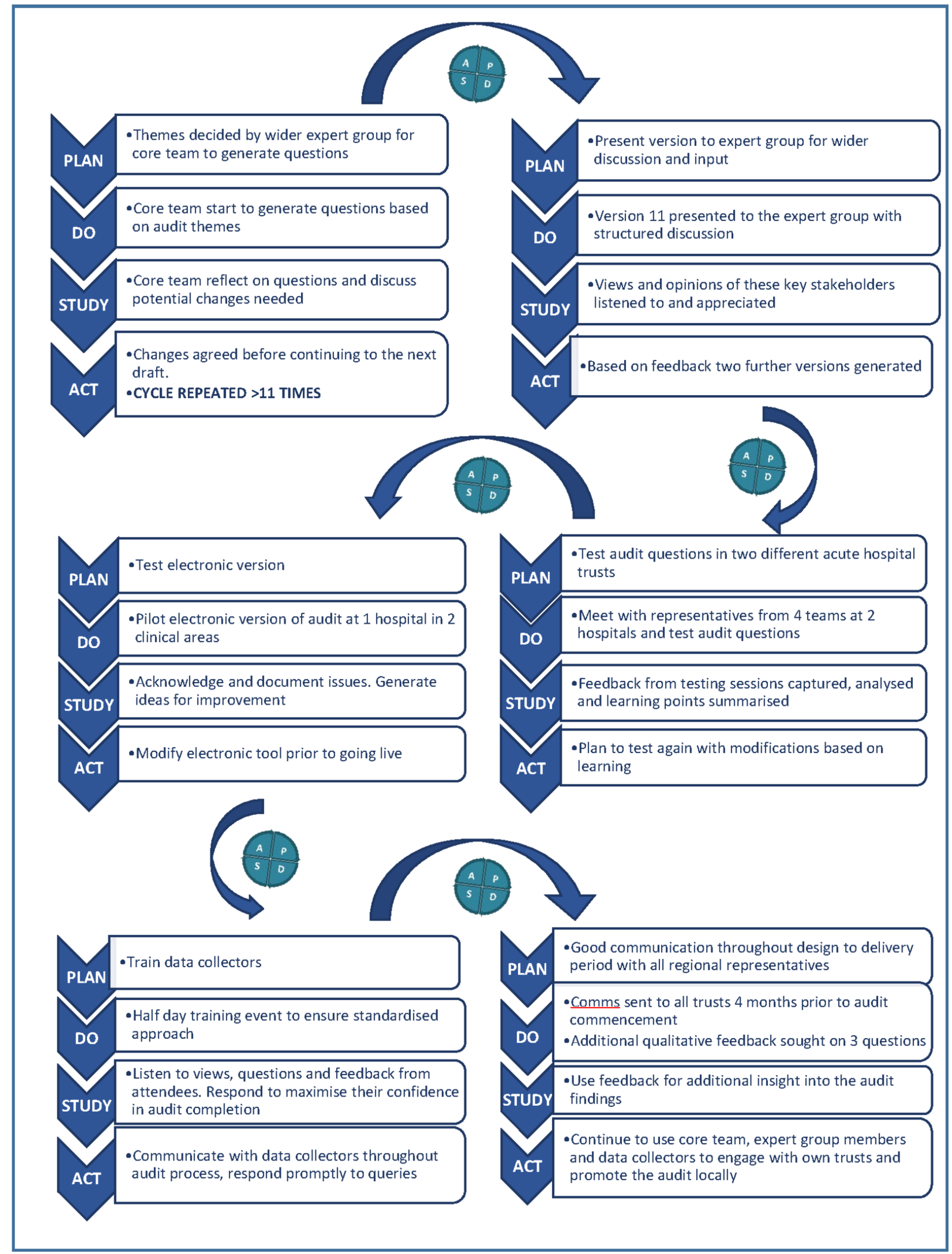

Figure 2 An illustration of the Plan-Do-Study-Act cycles. 
Initially, each meeting of the core group focused on a section topic, and the questions that would be required to demonstrate that organisations were meeting 'gold standards' for frailty in these areas. Between each meeting, expert group members reviewed these proposed questions while back in their base hospitals, considering whether they would be sufficient to both collect evidence of frailty work that was already ongoing, as well as highlight areas that could be lacking. Subsequent meetings were used to discuss these workplace 'studies' and tailor questions to ensure they were clear and would result in the capture of useful data.

\section{TESTING OF AUDIT}

Testing of audit was completed across two sites to test the feasibility of the questions in practice. Wording of the accompanying definitions document was altered as a consequence of this test. An online electronic survey tool was created for the audit. A unique feature of the online survey tool is the ability for multiple organisations to run the same survey to get answers to common questions or issues. The audit tool was built during July 2018 using the agreed audit questions; a flow diagram was developed to ensure that all questions held within the tool were captured for each specialty area. The electronic version was then tested in three clinical areas of one hospital. In addition to identifying some technical issues, we learnt that having senior clinicians from across the multidisciplinary team, for example, a nurse, allied health professional and medical doctor as opposed to non-clinical managers, provided optimal overview of the service.

\section{TRAINING DATA COLLECTORS}

Data collectors were trained to ensure consistency and provide internal validation to the data collection. This was a one off session that lasted 3 hours. Following from this event, changes to the definition document were made based on feedback received from the trainees.

\section{Ensuring good communication between the expert group, hospital clinical leads and data collector}

Each data collector contacted clinical leads from a ward area within each specialty to arrange a mutually convenient time to meet. They provided their clinical leads with an overview of the audit purpose before completing the audit with ward team members to capture a real-time snapshot of practice. This evidenced the variability in process across specialities. The audit questions were available via an electronic link. Discussing with team members in different specialities and allowing them to answer helped to eliminate bias and preconceptions of frailty experts.

Each organisation was provided with in-depth analysis of their audit outcomes; the high-level results were presented at a workshop on 26 February 2019 for medicine for older people clinical leads, hospital senior management, frailty experts representing acute and some community organisations from throughout the
Wessex region and three national clinical leads. The outputs were reviewed, the findings were interpreted and service improvement opportunities were explored. Delegates considered what the findings meant to future practice, resources and workforce. They were challenged to consider specific questions relating to each theme in a cross-regional perspective. Each hospital then met to discuss and prioritise their frailty action plans over the next 6 months, 12 months and 18 months.

Additional qualitative questions were asked: with regards to frailty provision, what are you doing well? What would you like to do next/more of? What are the barriers to achieving this? The narrative questions provided additional insight on where to focus subsequent QI projects.

\section{Findings}

Feedback from the whole expert group regarding question prioritisation resulted in the initial audit draft. Although members largely agreed on which questions should be a priority, there was also discussion around the intricacies of frailty and how each question could provide key information and insight into care provision. This resulted in the audit questions being expanded, with the view that even if organisations were not yet able to provide all services included in the audit, it could be used as the basis of a 'best practice model', not unlike SSNAP. ${ }^{5}$

Modifications: four hospitals were unable to release a representative to attend the training session. Two AHSN team members and the audit clinical coordinator visited the sites and worked alongside them using the electronic tool to support them to complete their data collection to ensure a consistent approach across all participating sites.

One hundred per cent of hospitals completed the Wessex Acute Frailty Audit: six acute hospitals, three district and one community with an acute medical receiving ward. Fifty-eight ward areas participated during October and November 2018. Outcomes from the online audit tool were analysed manually in Excel.

This paper has focused on the application of QI methodology to design and implement a multisite audit as opposed to the audit results; however, there are some key findings that have helped us move the work on further. No hospitals were achieving all elements of any section of the audit which, although expected, demonstrated we have no 'gold standard' service available in the region. Different areas showed huge variability both within different wards, within the same hospital and across the region. Key themes identified by the audit were a lack of awareness and frailty training outside of medicine for older people specialities, significant variability of frailty identification and whether and how CGA was carried out within individual trusts. Figure 3 outlines a sample of the response variations for two specific questions. The following are some high level results from each audit theme. 


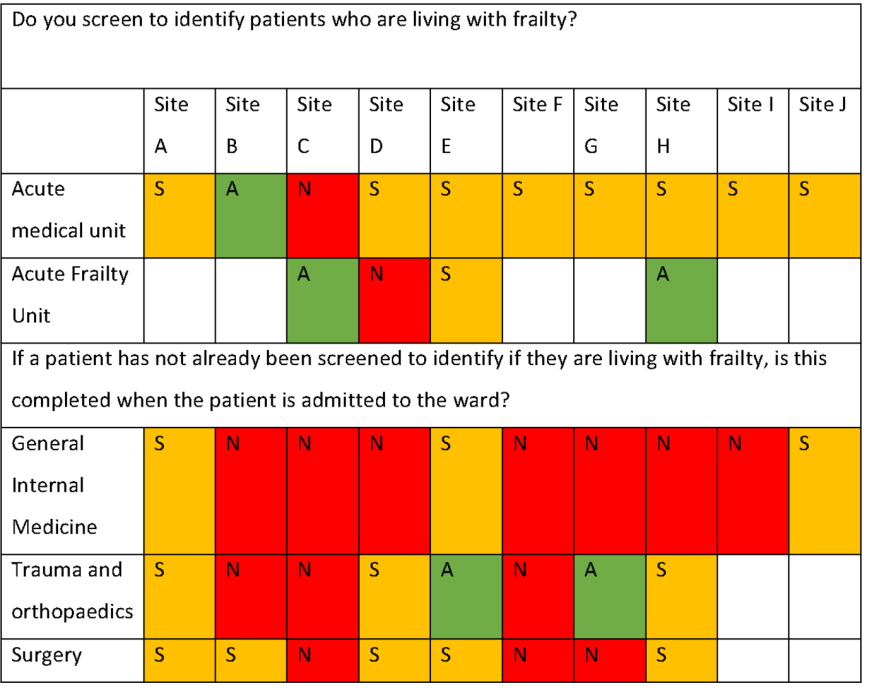

Figure 3 Identification of frailty by area and hospital. A, always; $\mathrm{N}$, never; $\mathrm{S}$, sometimes.

\section{Identification of frailty}

Acorss the region there is variability variability in frailty identification instruments used, where tools are used the Clinical Frailty Scale ${ }^{20}$ was most commonly applied. Once a person is identified as living with frailty, they are generally not 'flagged' in the patient records. Where they are documented (flagged) in the medical notes, the flag is not applied electronically, hospitals do not know where the people with frailty are in the hospitals making it difficult to target the required care. Access to primary care electronic frailty index/frailty scores is variable across hospital settings.

\section{Supporting people living with frailty in the hospital setting} Of the 58 wards audited, $30 \%$ always involve the person/ next of kin as standard practice during the discharge process. Outside of medicine for older people wards and some emergency departments, CGAs are not routinely carried out and rarely involve the person and/or those closest to them. Eighteen per cent of wards use a holistic assessment with a personalised problem list and frailty care plan developed to reflect the needs of the person. Most wards do not make reference to a frailty plan in the person's discharge summary when they leave hospital.

\section{FRAILTY TRAINING}

Frailty training is not mandatory in any of the hospitals audited. Any training that does occur is face to face, with examples of variability of workshops and lectures across hospitals. No formal records are kept so we are unable to identify who has completed.

\section{What are you doing well?}

In response to this question, one hospital stated that healthcare professionals are now more frailty aware than before with a good culture of case finding during morning medical handover as often a community geriatrician present. Another hospital conveyed they had well-established links to the community frailty support team. Although one hospital did not screen for frailty, they reported they were good at directing to Rapid Access Consultant Evaluation for CGA. There was evidence that having an older persons' assessment liaison team reduced length of stay and increased the proportion of CGAs completed. Establishing better community links and providing early supported discharge meant more assessments could be done with the person in their own home rather than in the hospital setting.

\section{What would you like to do next?}

Increased frailty training for the multidisciplinary team was a common theme for what people would like to do next in their organisations, "More training on frailty, changing people's mind sets to avoid admission and keep people living well at home'. Having a specialist frailty interprofessional team for those organisations that do not have them was a priority. Having and increased focus more resources on the community with a unified information technology system was identified as a method for sharing plans between organisations more efficiently in addition to follow-up after discharge, 'You are limited for what you can do. You put all the plans in place but are they being followed through?'. All organisations were keen to be involved with any regional wide QI projects as a result of the audit with one hospital wishing to focus on frailty recognition and management QI project in surgery.

\section{What are the barriers?}

In one organisation, a clinical lead (Geroatrican by profession) cited financial resources out with the hospital setting as being a barrier to change, 'More timely discharges could be made with investment in community services, outreach, care at home, intermediate care and social services'. Another geriatrician believes the barrier is generally other departments who do not want to upskill, 'They just want us to come and sort it out, and they seem to think we as geriatricians are walking Multi-disciplinary teams. I can understand that as I wouldn't be able to fix a hip so maybe I shouldn't expect an orthopaedic surgeon to do a CGA?'.

\section{DISCUSSION \\ Summary}

By engaging with key stake holders in each acute hospitals and using a QI framework, we collaboratively established a process for assessing processes of care for older people living with frailty. This has enabled the development of both regional and individual organisational level approaches to meeting the deficits of frailty provision and contributed to the sustainability of future projects.

\section{INTERPRETATION}

National audit has contributed to the improvement in quality of care for people with stroke with the data contributing to the understanding of the level of quality 
but also used by individual hospitals to develop QI initiatives over many years. ${ }^{21}$ The expectation is that this regional approach to frailty audit is the first step on a similar journey. Results from the audit were fed back to individual organisations to allow local action planning. The evidence gaps and variation within and across hospitals has identified opportunities for improvement particularly around consistent approaches to identification of those living with frailty, supporting those people living with frailty and the training of staff in frailty identification and management.

\section{LIMITATIONS}

The audit responses are based on the auditor and view of staff on the ward completing the audit. Data collectors were not formally assessed on their understanding of definitions or their ability to complete audit in their work places. Although this was implemented informally, all data collectors were senior clinicians working in the specialty, and therefore, it is assumed that they have the relevant knowledge to complete the task effectively.

\section{CONCLUSIONS}

Using QI methodology with a collaborative approach allowed the creation of a successful regional wide audit, highlighting areas of different practice and key focuses for improvement. This allowed subsequent development of bespoke hospital and Wessex regional level service improvement work streams, which can continue to use QI methods. The audit is now being adapted for the community setting based on the new primary care network structure. There has been national interest in the audit to be run in other areas of the country.

\section{NEXT STEPS}

The Wessex Acute Frailty Audit is to rerun in 2020 to establish trends in frailty care and impacts of work post2018 audit. Specialties not involved in the pilot audit will be incorporated; this will include older person's mental health, oncology, cardiology, renal and neurology. The expert group are ratifying draft standards for screening, identifying frailty within the acute setting, promoting the best approaches to sharing of frailty information and advocating the use of frailty flagging to enable appropriate information to be included in individual's discharge letters. A working group of 25 frailty experts have peer reviewed education materials and mapped to National Health Service England and Health Education England (2018) Tier 1 and 2 Skills for Health frailty competen$\mathrm{cies}^{22}$ to deliver a hospital-wide training approach. A local university is working with stakeholders to provide curriculum that meets Tier 3 standard. Structured evaluation of materials is planned for early 2020 across four organisations including social services. The development of frailty leaflets and posters will also form part of the educational toolkit for use.
A number of organisations are planning frailty education days during 2020 to promote the educational materials. Local organisations have developed QI projects that have the opportunity for learning and approaches to be shared across the region and will include representation of people living with frailty and those closest to them in the design and review of service improvement outputs.

Acknowledgements Thank you to all expert group members and data collectors. J Duffy, H Patel, C Wade Smith, E Maxwell, D Kryl, R Everett, P Collins, G Pratt, J Walker, B Windle, E Pugh, S Gladdish, S James, L Girling, J Richards, A Hassan, G Brittan, M Board, A McGuiness, G Hibberd and T Nivedika. Quality improvement coaching provided by N Davey at Quality Improvement Clinic, Health Education England South East, National Institute of Health Research Dissemination Centre, Basingstoke and North Hampshire County Hospital, Dorset County Hospital, Poole Hospital, Queen Alexandra Hospital, Royal Hampshire County Hospital, The Royal Bournemouth and Christchurch Hospital, Salisbury District Hospital, St Marys' Hospital, University Hospital Southampton and Lymington New Forest Hospital.

Contributors All authors made substantial contributions to conception and design, or acquisition of data, or analysis and interpretation of data. LAL was clinical coordinator for the project and was supervised by KW. KW, CS and KB were original core group members of the project from January 2018. CD became programme manager in October 2018. TC critiqued all versions of the manuscript and provided feedback that significantly contributed to the article development. Each of the authors were involved in revising the manuscript critically for important intellectual content. All authors have given final approval of the version to be published.

Funding This work was fully supported financially and resourced by the Wessex Academic Health Science Network. Each organisation contributed by allowing the time and expertise of the expert and core group members that was not resourced by the Wessex AHSN.

Competing interests None declared.

Patient consent for publication Not required.

Provenance and peer review Not commissioned; externally peer reviewed.

Data availability statement Data are available upon request.

Open access This is an open access article distributed in accordance with the Creative Commons Attribution Non Commercial (CC BY-NC 4.0) license, which permits others to distribute, remix, adapt, build upon this work non-commercially, and license their derivative works on different terms, provided the original work is properly cited, appropriate credit is given, any changes made indicated, and the use is non-commercial. See: http://creativecommons.org/licenses/by-nc/4.0/.

ORCID iD

Lucy Anne Lewis http://orcid.org/0000-0002-0520-9140

\section{REFERENCES}

1 Khandelwal D, Goel A, Kumar U, et al. Frailty is associated with longer hospital stay and increased mortality in hospitalized older patients. J Nutr Health Aging 2012;16:732-5.

2 Ertel KA, Glymour MM, Glass TA, et al. Frailty modifies effectiveness of psychosocial intervention in recovery from stroke. Clin Rehabil 2007;21:511-22.

3 NHS. Frailty resources. Available: https://www.england.nhs.uk/ ourwork/clinical-policy/older-people/frailty/frailty-resources/

4 Pilotto A, Panza F. Comprehensive geriatric assessment: evidence. Oxford TextBook of Geriatric Medicine, 2017.

5 The Sentinel Stroke National Audit Programme(SSNAP). Available: https://www.strokeaudit.org/About-SSNAP.aspx

6 National Institute Health Research Dissemination Centre. Themed review: Comprehensive Care - Older people with frailty in hospital, 2017. Available: https://www.dc.nihr.ac.uk/themed-reviews/frailty-inhospital-research.htm [Accessed Jun 2019].

7 Ellis G, Gardner M, Tsiachristas A, et al. Comprehensive geriatric assessment for older adults admitted to hospital: meta-analysis of randomised controlled trials, 2017. Available: https://www.ncbi.nIm. nih.gov/pubmed/28898390 [Accessed Jun 2019].

8 Fox MT, Sidani S, Persaud M, et al. Acute care for elders components of acute geriatric unit care: systematic descriptive review. J Am Geriatr Soc 2013;61:939-46. 
9 NICE. Transition between inpatient hospital settings and community or care home settings for adults with social care needs, 2015. Available: https://www.nice.org.uk/guidance/ng27 [Accessed Jun 2019].

10 Charles AW, Kershaw M, Ham C, et al. Year of integrated care systems: reviewing the journey the kings fund London 2018.

11 Roberts H, Conroy SP. Hospital wide CGA. Available: https://www. bgs.org.uk/resources/hospital-wide-comprehensive-geriatricassessment-how-cga-history-of-the-project [Accessed Jun 2019].

12 Ellis G, Whitehead MA, Robinson D, et al. Comprehensive geriatric assessment for older adults admitted to hospital: meta-analysis of randomised controlled trials. BMJ 2011;343:d6553.

13 Craig P, Dieppe P, Macintyre S, et al. Medical Research Council guidance. developing and evaluating complex interventions: the new medical Research Council guidance. Br Med J Sep 2008;29:a165.

14 Banerjee J, V O'Leary KS.C. The silver book: quality care for older people with urgent and emergency care needs. The British Geriatric Society, 2012.

15 Langley GJ. The improvement guide: a practical approach to enhancing organizational performance. John Wiley \& Sons, 2009.
16 Hillman T, Roueche A. Quality improvement. BMJ 2011;342:d2060.

17 Narine L, Persaud DD. Gaining and maintaining commitment to large-scale change in healthcare organizations. Health Serv Manage Res 2003:16:179-87.

18 Braithwaite J, Churruca K, Long JC, et al. When complexity science meets implementation science: a theoretical and empirical analysis of systems change. BMC Med 2018;16:63.

19 Davey N. Action on audit: injecting a few PDSAs to deliver service improvement, 2017. Available: https://t.co/yl9ZRjVm82 [Accessed 4th Sep 2018].

20 Rockwood K, Song X, MacKnight C. A global clinical measure of fitness and frailty in elderly people. Can Med Assoc J 2005;173:489-95.

21 King's College London. Sentinel stroke national audit programme (SSNAP) clinical audit April 2013 - March 2018 annual public report 2019.

22 NHS England and Health Education England. Frailty core capabilities framework, 2018. Available: https://www.skillsforhealth.org.uk/ services/item/607-frailty-core-capabilities-framework 